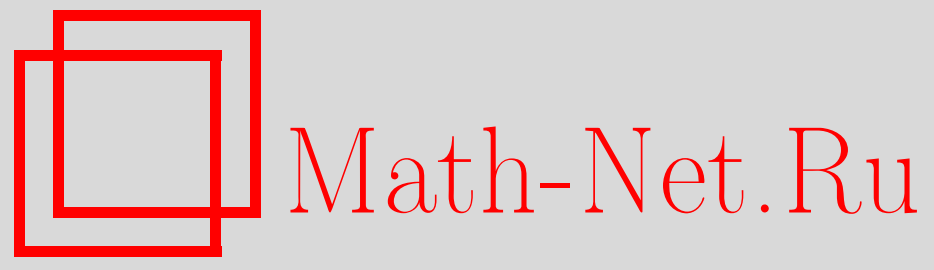

А. А. Буханько, Условие пластичности, связанное с линиями уровня поверхности деформационных состояний, и особенности его приложения в теории идеальной пластичности, Вестн. Сам. гос. техн. ун-та. Сер. Физ.-мат. науки, 2013, выпуск 1(), 199-206

DOI: https://doi.org/10.14498/vsgtu1172

Использование Общероссийского математического портала Math-Net.Ru подразумевает, что вы прочитали и согласны с пользовательским соглашением

http://www.mathnet.ru/rus/agreement

Параметры загрузки:

IP : 34.229 .45 .116

26 апреля 2023 г., $13: 30: 52$

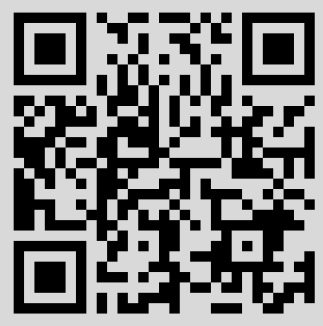




\title{
УСЛОВИЕ ПЛАСТИЧНОСТИ, СВЯЗАННОЕ С ЛИНИЯМИ УРОВНЯ ПОВЕРХНОСТИ ДЕФОРМАЦИОННЫХ СОСТОЯНИЙ, И ОСОБЕННОСТИ ЕГО ПРИЛОЖЕНИЯ В ТЕОРИИ ИДЕАЛЬНОЙ ПЛАСТИЧНОСТИ
}

\author{
А. А. Буханъко \\ Самарский государственный аэрокосмический университет им. ак. С. П. Королёва \\ (национальный исследовательский университет), \\ 443086, Россия, Самара, Московское ш., 34. \\ E-mail: abukhanko@mail.ru
}

\begin{abstract}
Рассматривается особенность построения решения краевых задач в теории плоской деформации идеалъного жёсткопластического тела при условии пластичности, связанном с линиями уровня поверхности деформационных состояний упрочняющегося несжимаемого жёсткопластического тела.
\end{abstract}

Ключевые слова: пластичность, поверхность деформационных состояний, жёсткопластическое тело.

Введение. Традиционно приложением теории пластичности считаются задачи обработки материалов давлением. С другой стороны, приложение теории пластического течения к задачам механики разрушения остается недостаточно изученным. С точки зрения механики разрушения процесс разрушения пластических тел состоит из двух этапов: достижения материалом предельного состояния (зарождение трещины) и образования новых свободных поверхностей (распространение трещины) [1]. Если первый процесс обусловлен рассеянием работы внутренних сил на пластических деформациях, связанных с упрочнением материала, вызывающих его повреждение, то второй процесс происходит без упрочнения материала в связи с исчерпанием его пластических свойств. Условия второго этапа разрушения позволяют поставить задачу об определении пластического течения материала в условиях его предельного упрочнения при идеально пластических свойствах.

\section{1. Поверхность нагружения, связанная с линиями уровня поверхности де-} формационных состояний. Если рассматривать несжимаемое упрочняющееся изотропное жёсткопластическое тело, то все его деформационные состояния будут лежать на гиперболической поверхности третьего порядка в пространстве главных деформаций, рис. 1, а [2,3]. Все деформационные процессы описываются линиями $L$ на этой поверхности или их проекциями $l$ на девиаторной плоскости, рис. 1, б. Вид линий на поверхности зависит от истории нагружения. В частности, ортогональным линиям будет соответствовать одноосное деформирование цилиндрического образца.

Наиболее простой линией, характеризующей предельное состояние материала, является сечение поверхности $\Sigma$ плоскостями, параллельными девиаторной плоскости. Далее будем называть эти линии «линиями уровня», рис. 1, б. Эти линии имеют вид замкнутого криволинейного треугольника с тремя осями симметрии [4-6].

Связь между поверхностью нагружения и предельным состоянием мате-

Анастасия Андреевна Буханъко (к.ф.-м.н., доцент), докторант, каф. высшей математики. 


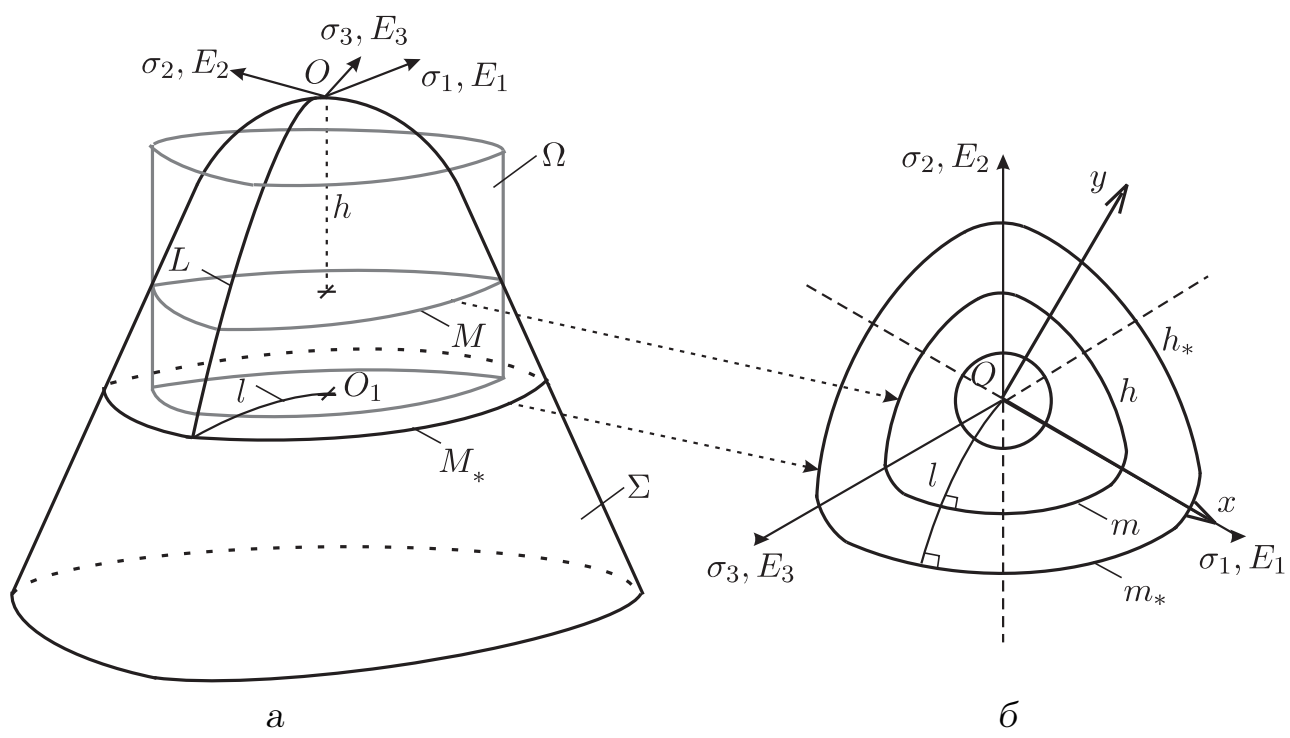

Рис. 1. Поверхности деформационных состояний $\Sigma$ и нагружения $\Omega$ (а) и линии уровня (б)

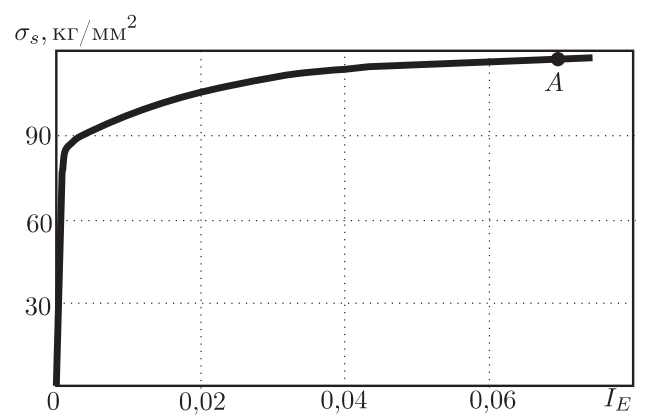

Рис. 2. Диаграмма нагружения для сплава ЭК79

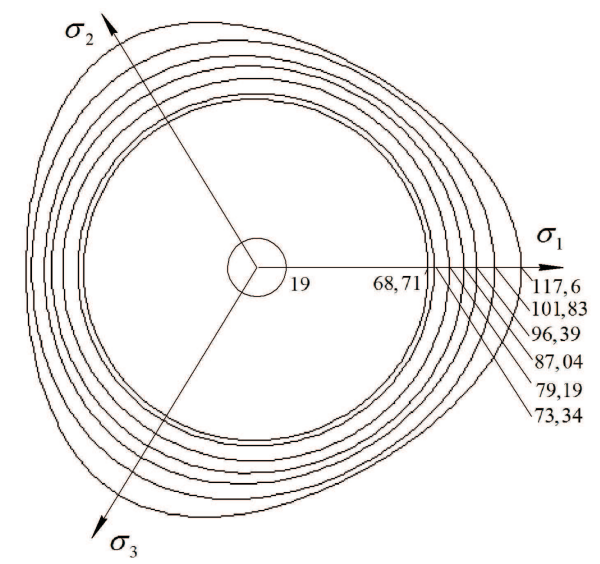

Рис. 3. Проекции поверхности нагружения на девиаторную плоскость для сплава ЭК79 в пространстве главных напряжений 
риала определяется гипотезой [7]: предельным состоянием материала считается состояние исчерпания его пластических свойств, т.е. состояние предельного упрочнения. Предполагаем, что при определенном уровне деформирования условие пластичности определяется формой линии уровня, размер которой соответствует диаграмме нагружения для конкретного материала. Для того чтобы связать поверхность деформационных состояний $\Sigma$ и поверхность нагружения $\Omega$, необходимо перестроить диаграмму нагружения (см. рис. 2): предполагается использование гипотезы единой кривой, но построенной не в традиционных координатах интенсивностей касательных напряжений и деформаций сдвига, а в виде зависимости текущего значения предела текучести $\sigma_{S}\left(I_{E}\right)$, определяемого значением параметра упрочнения, который совпадает с модулем первого инварианта $I_{E}$ тензора конечных деформаций Альманси. Точке $A$ предельного состояния материала на диаграмме нагружения соответствует некоторая линия $M$ на предельной поверхности или $m$ на девиаторной плоскости. Причем, если материал монотонно однократно деформировался, то положение предельной линии будет максимально удалено от точки $O$ недеформированного состояния. Если же материал испытывал сложное нагружение (включая циклическое с произвольной формой циклов), то линия предельного состояния примет другое положение, ближе к недеформированному состоянию, согласно известной формуле Коффина-Мэнсона [8].

Если рассматривать последовательность сечений поверхности деформационных состояний плоскостями, параллельными девиаторной плоскости, то форма кривой текучести изменяется следующим образом: при приближении к недеформированному состоянию она будет стремиться к окружности, т.е. к условию пластичности Мизеса. При удалении от этой точки она будет принимать все более треугольнообразную форму, которая в пределе будет стремиться к треугольнику. Переход с одной линии уровня на другую происходит при одной и той же мощности работы внутренних сил для всех ортогональных процессов деформирования, которая совпадает с удельной мощностью работы внутренних сил при одноосном растяжении цилиндрического образца.

На рис. 3 представлены проекции поверхности нагружения на девиаторную плоскость для сплава ЭК79 в пространстве главных напряжений.

Таким образом, при рассмотрении задач деформирования упрочняющегося жёсткопластического тела на каждом уровне деформаций для определения компонент тензора скорости деформации $\varepsilon_{i j}$ может быть поставлена задача теории идеальной пластичности при заданном параметре упрочнения, характеризующем положение поверхности нагружения. За параметр упрочнения выбирается модуль первого инварианта тензора конечных деформаций Альманси $h=\left|I_{E}\right|$.

2. Формы записи условия пластичности. В работах $[2,3]$ уравнение линий уровня было представлено в виде системы уравнений, геометрически представляющей собой пересечение двух поверхностей:

$$
\left\{\begin{array}{l}
C_{1} C_{2} C_{3}=1, \\
H=\frac{1}{\sqrt{3}}\left(C_{1}+C_{2}+C_{3}\right) .
\end{array}\right.
$$

Здесь $C_{i}=1-2 E_{i}$ - компоненты тензора деформаций Коши, $E_{i}-$ компонен- 
ты тензора конечных деформаций Альманси.

Условие пластичности (1) может быть представлено в симметричном (относительно перестановок $\left.\left(\sigma_{1}, \sigma_{2}, \sigma_{3}\right),\left(\sigma_{2}, \sigma_{3}, \sigma_{1}\right),\left(\sigma_{3}, \sigma_{1}, \sigma_{2}\right)\right)$ виде:

в компонентах тензора напряжений:

$$
\begin{gathered}
\left(\sigma_{2}+\sigma_{3}-2 \sigma_{1}\right)\left(\sigma_{3}+\sigma_{1}-2 \sigma_{2}\right)\left(\sigma_{1}+\sigma_{2}-2 \sigma_{3}\right)+ \\
+\frac{9}{2} \sigma_{S}\left(I_{E}\right)\left[\left(\sigma_{1}-\sigma_{2}\right)^{2}+\left(\sigma_{2}-\sigma_{3}\right)^{2}+\left(\sigma_{3}-\sigma_{1}\right)^{2}\right]= \\
=27 \sigma_{S}^{3}\left(I_{E}\right)\left[1-\left(\frac{3}{3-2 I_{E}}\right)^{3}\right]
\end{gathered}
$$

в компонентах девиатора напряжений:

$$
s_{1} s_{2} s_{3}+\sigma_{S}\left(I_{E}\right)\left(s_{1} s_{2}+s_{2} s_{3}+s_{3} s_{1}\right)=\sigma_{S}^{3}\left(I_{E}\right)\left[\left(\frac{3}{3-2 I_{E}}\right)^{3}-1\right]
$$

или

$$
I I I_{s}-\sigma_{S}\left(I_{E}\right) I I_{s}=\sigma_{S}^{3}\left(I_{E}\right)\left[\left(\frac{3}{3-2 I_{E}}\right)^{3}-1\right]
$$

где $\sigma_{S}$ - предел текучести, $I_{E}=E_{1}+E_{2}+E_{3}-$ первый инвариант тензора конечных деформаций Альманси. При этом значения $\sigma_{S}=\sigma_{S}\left(I_{E}\right)$ определяются из эксперимента на одноосное растяжением образца и характеризуют состояние упрочнения на данном уровне деформаций, определяемом параметром упрочнения $h=\left|I_{E}\right|$.

Отметим, что в общем случае новое условие пластичности содержит второй и третий инварианты тензора напряжений:

$$
f\left(I I_{\Sigma}, I I I_{\Sigma}\right)=K
$$

где $K$ - некоторая константа, характеризующая состояние материала. При выбранном условии пластичности выполняется условие несжимаемости, но не выполняется условие пропорциональности компонент тензора скорости деформации и девиатора тензора напряжений. Рассмотрим на примере плоской деформации.

3. Особенности условия пластичности при плоской деформации. В рамках плоской деформации теории идеального жёсткопластического тела условие (2) принимает следующий вид:

$$
\begin{aligned}
& {\left[\left(\sigma_{y}+\sigma_{z}-2 \sigma_{x}\right)\left(\sigma_{z}+\sigma_{x}-2 \sigma_{y}\right)-9 \tau_{x y}^{2}\right]\left(\sigma_{x}+\sigma_{y}-2 \sigma_{z}\right)+} \\
& +\frac{9}{2} \sigma_{S}\left(I_{E}\right)\left[\left(\sigma_{x}-\sigma_{y}\right)^{2}+\left(\sigma_{y}-\sigma_{z}\right)^{2}+\left(\sigma_{z}-\sigma_{x}\right)^{2}+6 \tau_{x y}^{2}\right]= \\
& \quad=27 \sigma_{S}^{3}\left(I_{E}\right)\left[1-\left(\frac{3}{3-2 I_{E}}\right)^{3}\right]
\end{aligned}
$$

Здесь условие рассматривается как не зависящее от дальнейших деформаций на данном уровне упрочнения, определяемом величиной $I_{E}$ (например, при состоянии предельного упрочнения). 
Ассоциированный закон пластического течения

$$
\varepsilon_{i j}=\lambda \frac{\partial f}{\partial \sigma_{i j}}
$$

и условие (3) позволяют определить компоненты тензора скорости деформаций:

$$
\begin{aligned}
& \varepsilon_{x}=\lambda \frac{\partial f}{\partial \sigma_{x}}=3 \lambda\left\{\left[2\left(\sigma_{y}-\sigma_{z}\right)^{2}-\left(\sigma_{x}-\sigma_{y}\right)^{2}-\left(\sigma_{z}-\sigma_{x}\right)^{2}-3 \tau_{x y}^{2}\right]+\right. \\
& \left.+3 \sigma_{S}\left(I_{E}\right)\left[2 \sigma_{x}-\sigma_{y}-\sigma_{z}\right]\right\} ; \\
& \varepsilon_{y}=\lambda \frac{\partial f}{\partial \sigma_{y}}=3 \lambda\left\{\left[2\left(\sigma_{z}-\sigma_{x}\right)^{2}-\left(\sigma_{y}-\sigma_{z}\right)^{2}-\left(\sigma_{x}-\sigma_{y}\right)^{2}-3 \tau_{x y}^{2}\right]+\right. \\
& \left.+3 \sigma_{S}\left(I_{E}\right)\left[2 \sigma_{y}-\sigma_{z}-\sigma_{x}\right]\right\} ; \\
& \varepsilon_{z}=\lambda \frac{\partial f}{\partial \sigma_{z}}=3 \lambda\left\{\left[2\left(\sigma_{x}-\sigma_{y}\right)^{2}-\left(\sigma_{z}-\sigma_{x}\right)^{2}-\left(\sigma_{y}-\sigma_{z}\right)^{2}+6 \tau_{x y}^{2}\right]+\right. \\
& \left.+3 \sigma_{S}\left(I_{E}\right)\left[2 \sigma_{z}-\sigma_{x}-\sigma_{y}\right]\right\} ; \\
& \frac{1}{2} \eta_{x y}=\lambda \frac{\partial f}{\partial \tau_{x y}}=9 \lambda \tau_{x y}\left[2 \sigma_{z}-\sigma_{x}-\sigma_{y}+3 \sigma_{S}\left(I_{E}\right)\right] .
\end{aligned}
$$

Согласно (4) при выбранном условии пластичности (3) в случае плоской деформации выполняются:

условие несжимаемости:

$$
\varepsilon_{1}+\varepsilon_{2}+\varepsilon_{3}=\lambda \frac{\partial f}{\partial \sigma_{1}}+\lambda \frac{\partial f}{\partial \sigma_{2}}+\lambda \frac{\partial f}{\partial \sigma_{3}}=0
$$

условие соосности тензора скорости деформации и девиатора напряжений:

$$
\frac{\varepsilon_{x}-\varepsilon_{y}}{\eta_{x y}}=\frac{\frac{\partial f}{\partial \sigma_{x}}-\frac{\partial f}{\partial \sigma_{y}}}{2 \frac{\partial f}{\partial \tau_{x y}}}=\frac{\sigma_{x}-\sigma_{y}}{2 \tau_{x y}}=\frac{s_{x}-s_{y}}{2 \tau_{x y}} .
$$

Однако из условия (5) и соотношений (4) не следует пропорциональность тензора скорости деформации и девиатора напряжений $\varepsilon_{i j} \neq \lambda s_{i j}$, т.е. не выполняются уравнения теории пластичности Сен-Венана-Мизеса.

Подставляя в условие (3) и третье соотношения (4) при $\varepsilon_{z}=0$ известные формулы теории напряжений:

$$
\left\{\begin{array}{l}
\sigma_{x}=p+q \cos 2 \psi \\
\sigma_{y}=p-q \cos 2 \psi \\
\tau_{x y}=q \sin 2 \psi
\end{array}\right.
$$


где

$$
p=\frac{\sigma_{1}+\sigma_{2}}{2}, \quad q=\frac{\sigma_{1}-\sigma_{2}}{2},
$$

$\psi$ - угол между первым главным напряжением и осью $x$, получим систему уравнений:

$$
\left\{\begin{array}{c}
{\left[\left(\sigma_{z}-p\right)^{2}-9 q^{2}\right]\left(\sigma_{z}-p\right)-\frac{9}{2} \sigma_{S}\left(I_{E}\right)\left[\left(\sigma_{z}-p\right)^{2}+3 q^{2}\right]=} \\
=\frac{27}{2} \sigma_{S}^{3}\left(I_{E}\right)\left[\left(\frac{3}{3-2 I_{E}}\right)^{3}-1\right], \\
\left(\sigma_{z}-p\right)^{2}-3 \sigma_{S}\left(I_{E}\right)\left(\sigma_{z}-p\right)=3 q^{2},
\end{array}\right.
$$

согласно которой имеем приведенное уравнение относительно $\left(\sigma_{z}-p\right)$ :

$$
\left(\sigma_{z}-p\right)^{3}-\frac{27}{4} \sigma_{S}^{2}\left(I_{E}\right)\left(\sigma_{z}-p\right)-\frac{27}{4} \sigma_{S}^{3}\left(I_{E}\right)\left[1-\left(\frac{3}{3-2 I_{E}}\right)^{3}\right]=0 .
$$

Дискриминант уравнения (6) всегда отрицателен, поэтому оно имеет три действительных решения, согласно которым получаем значения третьего главного напряжения:

$$
\left(\sigma_{z}\right)_{1,2,3}=\frac{1}{2}\left(\sigma_{x}+\sigma_{y}\right)+3 \sigma_{S}\left(I_{E}\right) \cos \left(\frac{\varphi}{3}+\gamma_{1,2,3}\right),
$$

где

$$
\varphi=\arccos \left[1-\left(\frac{3}{3-2 I_{E}}\right)^{3}\right], \quad \gamma_{1}=0, \quad \gamma_{2}=\frac{2 \pi}{3}, \quad \gamma_{3}=\frac{4 \pi}{3} .
$$

При приближении к недеформированному состоянию имеем

$$
\lim _{I_{E} \rightarrow 0}\left(\sigma_{z}\right)_{1,2}=\frac{1}{2}\left(\sigma_{x}+\sigma_{y}\right) \pm \frac{3 \sqrt{3}}{2} \sigma_{S}\left(I_{E}\right), \quad \lim _{I_{E} \rightarrow 0}\left(\sigma_{z}\right)_{3}=\frac{1}{2}\left(\sigma_{x}+\sigma_{y}\right) .
$$

Заметим, что если $\sigma_{S} \rightarrow 0$ при $I_{E} \rightarrow 0$, то все три значения $\sigma_{z} \rightarrow \frac{1}{2}\left(\sigma_{x}+\sigma_{y}\right)$.

Подставляя (7) в условие (3), получим условие пластичности при условии плоской деформации в следующем виде:

$$
\left(\sigma_{x}-\sigma_{y}\right)^{2}+4 \tau_{x y}^{2}=\frac{4 \sigma_{S}^{2}\left(I_{E}\right)\left[\left(3-2 I_{E}\right)^{3}(\cos \Phi-1)^{2}(2 \cos \Phi+1)-27\right]}{\left(3-2 I_{E}\right)^{3}(2 \cos \Phi+1)}
$$

где

$$
\Phi=\frac{\varphi}{3}+\gamma_{1,2,3}
$$

Очевидно, что вид нового условия пластичности (8) при плоской деформации совпадает с известным условием Мизеса, отличие заключается в определении третьего главного значения тензора напряжений. Таким образом, дальнейший алгоритм решения задач плоской деформации идеального жёсткопластического тела совпадает с теорией плоской деформации при условии текучести Мизеса. 
Заключение. Показано, что при использовании нового условия пластичности в рамках плоской деформации теории идеального жёсткопластического тела выполняются условия несжимаемости и соосности тензора скорости деформации и девиатора напряжений, но не выполняется условие пропорциональности их компонент; алгоритм решения задач совпадает с алгоритмом решения задач при условии Мизеса, но приводит к новой формуле определения третьего главного значения тензора напряжений.

Работа выполнена при поддержке РФФИ (проект № 12-01-31283-мол_а).

\section{БИБЛИОГРАФИЧЕСКИЙ СПИСОК}

1. А. И. Хромов, А. А. Буханъко, О. В. Козлова, С. Л. Степанов, "Пластические константы разрушения" // ПМТФ, 2006. Т. 47, №2. С. 147-155; англ. пер.: A. I. Khromov, A. A. Bukhan'ko, O. V. Kozlova, S. L. Stepanov, "Plastic constants of fracture" // J. Appl. Mech. Tech. Phys., 2006. Vol. 47, no. 2. Pp. 274-281.

2. А. И. Хромов, Е. П. Кочеров, А. Л. Григоръева, “Деформационные состояния и условия разрушения жёсткопластических тел” // Докл. Акад. наук, 2007. Т. 413, № 4. С. 481-485; англ. пер.: A. I. Khromov, E. P. Kocherov, A. L. Grigor'eva, "Strain states and fracture conditions for rigid-plastic bodies" // Dokl. Phys., 2007. Vol. 52, no. 4. Pp. 228-232.

3. А. А. Буханъко, А. Л. Григоръева, Е. П. Кочеров, А. И. Хромов, "Деформационноэнергетический критерий разрушения жёсткопластических тел"// Изв. РАН. MTT, 2009. №6. С. 178-186; англ. пер.: A. A. Bukhan'ko, A. L. Grigor'eva, E. P. Kocherov, A. I. Khromov, "Strain-energy failure criterion for rigid-plastic bodies" // Mech. Solids, 2009. Vol. 44, no. 6. Pp. 959-966.

4. Г. С. Писаренко, А. А. Лебедев, "О форме предельной поверхности механического критерия прочности" // Прикл. механика, 1968. Т. 4, № 3. С. 45-50; англ. пер.: G. S. Pisarenko, A. A. Lebedev, "Shape of the limiting surface of a mechanical strength criterion" // Sov. Appl. Mech., 1968. Vol. 4, no. 3. Pp. 27-30.

5. И. И. Голъденблат, В. А. Копнов, "Общая теория критериев прочности изотропных и анизотропных материалов" // Пробл. прочн., 1971. №2. С. 65-69; англ. пер.: I. I. Gol'denblat, V. A. Kopnov, "General theory of criteria of strength for isotropic and anisotropic materials" // Strength of Materials, 1971. Vol. 3, no. 2. Pp. 184-188.

6. Г. А. Гениев, В. Н. Киссюк, Г. А. Тюпин, Теория пластичности бетона и железобетона. М.: Стройиздат, 1974. 316 c. [G. A. Geniev, V. N. Kissyuk, G. A. Tyupin, The Theory of Plasticity of Concrete and Reinforced Concrete. Moscow: Stroiizdat, 1974. 316 pp.]

7. Г. С. Писаренко, Н. С. Можаровский, Е. А. Антипов, Сопротивление жаропрочных материалов нестационарным силовым и температурным воздействиям. Киев: Наукова думка, 1974. 200 с. [G. S. Pisarenko, N. S. Mozharovskii, E. I. Antipov, Strength of heatresistant materials with respect to transient dynamic and thermal action. Kiev: Naukova Dumka, 1974. 200 pp.]

8. Е. П. Кочеров, А. А. Буханъко, А. И. Хромов, “Деформационно-энергетический подход и малоцикловая усталость материалов" // Вестник СГАУ, 2011. №3. Ч. 1. С. 23-27. [E. P. Kocherov, A. A. Bukhan'ko, A. I. Khromov, "Strain-energy approach and low-cycle fatigue of materials" // Vestnik SGAU, 2011. no. 3. Part 1. Pp. 23-27].

Поступила в редакцию 15/XI/2012; в окончательном варианте - 27/I/2013. 
MSC: 74C20

PLASTICITY CONDITION RELATED WITH LEVEL LINES OF STRAIN STATES SURFACE, AND FEATURES OF ITS APPLICATION IN THE IDEAL PLASTICITY THEORY

\section{A. A. Bukhanko}

S. P. Korolyov Samara State Aerospace University

(National Research University),

34, Moskovskoe sh., Samara, 443086, Russia.

E-mail: abukhanko@mail.ru

The feature of solution formation of boundary value problems is considered in plane strain of ideal rigid-plastic body when the plasticity condition is related with level lines of strain states surface of work-hardening incompressible rigid-plastic body.

Key words: plasticity, strain states surface, rigid-plastic body.

Original article submitted 15/XI/2012; revision submitted 27/I/2013.

Anastasia A. Bukhanko (Ph. D. (Phys. \& Math.)), Doctoral Candidate, Dept. of Higher Mathematics. 